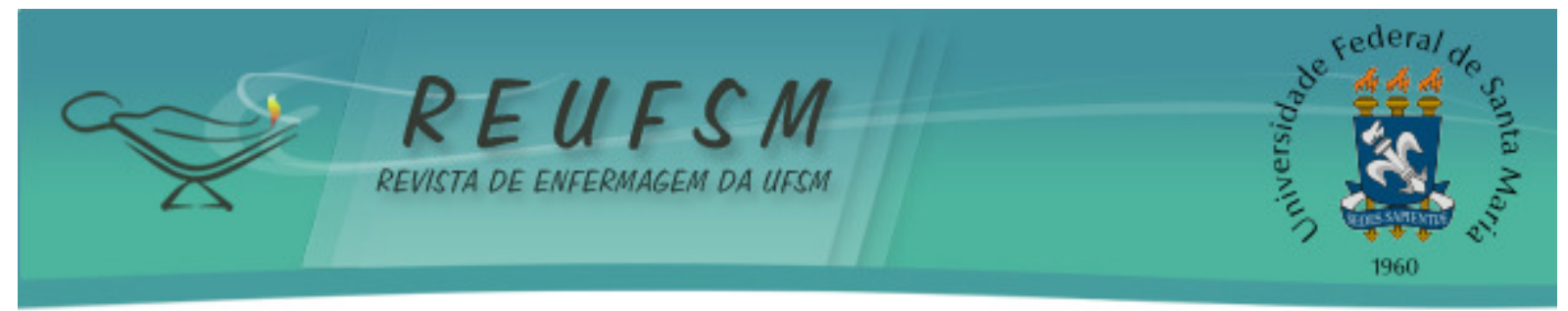

ARTIGO ORIGINAL

\title{
SUICÍDIO NA VOZ DE PROFISSIONAIS DE ENFERMAGEM E ESTRATÉGIAS DE INTERVENÇÃO DIANTE DO COMPORTAMENTO SUICIDA
}

\section{SUICIDE IN NURSING PROFESSIONAL VOICE AND INTERVENTION STRATEGIES BEFORE THE SUICIDAL BEHAVIOR}

\section{SUICIDIO EN LA VOZ DE PROFESIONALES DE LA ENFERMERÍA Y ESTRATEGIAS DE INTERVENCIÓN ANTE LA CONDUCTA SUICIDA}

\author{
Nara Reisdorfer ${ }^{1}$ \\ Graciela Machado de Araujo ${ }^{2}$ \\ Leila Mariza Hildebrandt ${ }^{3}$ \\ Taciana Raquel Gewehr ${ }^{4}$ \\ Janaine Nardino ${ }^{5}$ \\ Marinês Tambara Leite ${ }^{6}$
}

Doi: $10.5902 / 2179769216790$

RESUMO: Objetivo: analisar o conhecimento e as estratégias de intervenção de profissionais de enfermagem de um hospital geral, acerca do comportamento suicida. Método: pesquisa qualitativa e descritiva, realizada com 10 profissionais de um hospital geral. Para a coleta de dados foi utilizada a entrevista semiestruturada e a análise dos dados seguiu os passos da análise temática. Resultados: foram agrupados em dois temas, sendo que o primeiro versa sobre a concepção dos profissionais sobre suicídio e o segundo discute as intervenções junto a pessoas que tentaram ou com risco para o suicídio. Considerações finais: a equipe de enfermagem apresenta fragilidade no conhecimento sobre o comportamento suicida o que repercute nas intervenções realizadas. Para tanto, destaca-se a importância e necessidade de qualificação desses profissionais no intuito de proporcionar cuidados efetivos a pessoas com risco ou tentativa de suicídio.

Descritores: Enfermagem; Cuidados de Enfermagem; Suicídio; Tentativa de Suicídio.

ABSTRACT: Aim: to analyze nursing professionals' understanding and intervention strategies, in a general hospital, on suicidal behavior. Method: qualitative and descriptive study, performed with 10 professionals from a general hospital. Semi-structured interviews were used for data collection and the data analysis followed the steps of thematic analysis. Results: the results were grouped in two themes, the first refers to the professionals' conception about suicide while the second discusses the interventions concerning the people who attempted or at risk for suicide. Final remarks: the nursing

\footnotetext{
1 Acadêmica do Curso de Enfermagem da Universidade Federal de Santa Maria, Campus de Palmeira das Missões. Bolsista PET Enfermagem/Campus Palmeira das Missões/Rio Grande do Sul/Brasil. E-mail: nara.reisdorfer@hotmail.com

2 Acadêmica do Curso de Enfermagem da Universidade Federal de Santa Maria, Campus de Palmeira das Missões/RS. Bolsista PET REDES, Campus Palmeira das Missões/Rio Grande do Sul/Brasil. E-mail: gra_m_a@hotmail.com

${ }^{3}$ Enfermeira, Doutora em Ciências, Docente do Curso de Enfermagem da Universidade Federal de Santa Maria, Campus de Palmeira das Missões/ Campus Palmeira das Missões/Rio Grande do Sul/Brasil. E-mail: leilahildebrandt@yahoo.com.br

${ }^{4}$ Enfermeira, egressa do Curso de Enfermagem da Universidade Federal de Santa Maria, Campus de Palmeira das Missões/Campus Palmeira das Missões/Rio Grande do Sul/Brasil. E-mail: tacianaraquel@hotmail.com

${ }^{5}$ Enfermeira, Especialista em Gestão da Organização Pública de Saúde. Universidade Federal de Santa Maria, Campus de Palmeira das Missões/Rio Grande do Sul/Brasil. E-mail: jana.enfer07@yahoo.com.br

${ }^{6}$ Enfermeira, Doutora em Gerontologia Biomédica, Docente do Curso de Enfermagem da Universidade Federal de Santa Maria, Campus de Palmeira das Missões. Tutora do Grupo PET Enfermagem/Campus Palmeira das Missões/Rio Grande do Sul/Brasil. E-mail. tambaraleite@yahoo.com.br
} 


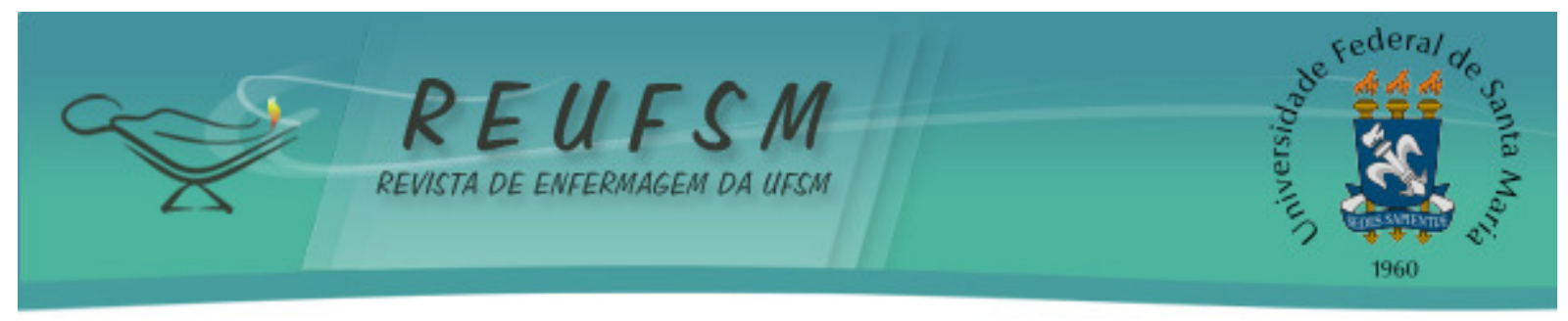

staff has weakness in knowledge of suicidal behavior which results in the interventions. This fact highlights the importance of the need for qualification of these professionals in order to provide effective care to people at risk or that has attempted suicide.

Descriptors: Nursing; Nursing Care; Suicide; Attempted Suicide.

RESUMEN: Objetivo: analizar los conocimientos y estrategias de intervención de profesionales de enfermería, en un hospital general, sobre la conducta suicida. Método: estudio cualitativo y descriptivo, con 10 profesionales de un hospital general. En la recolección de datos fue utilizada la entrevista semiestructurada y el análisis de los dados fue temático. Resultados: se agruparon en dos temas, lo primero se ocupa de la concepción de los profesionales sobre el suicidio y la segunda analiza las intervenciones con personas que han intentado suicidarse o en riesgo de suicidio. Consideraciones finales: el equipo de enfermería presenta dificultad en el conocimiento de la conducta suicida, lo que repercute en las intervenciones realizadas. Se destaca la importancia de la necesidad de capacitación de estos profesionales con el fin de proporcionar una atención eficaz a las personas en situación de riesgo o intento de suicidio.

Descriptores: Enfermería; Atención de Enfermería; Suicidio; Intento de suicídio.

\section{INTRODUÇÃO}

O suicídio apresenta-se como um fenômeno complexo e multicausal, fruto da interação de fatores de ordem filosófica, antropológica, psicológica, biológica e social. A consumação do suicídio deriva de um ato provocado e levado a cabo pelo indivíduo, com intenção clara de pôr fim à vida. ${ }^{1}$ Para a Organização Mundial da Saúde (OMS), cerca de 800 mil pessoas se suicidam por ano em todo o planeta, uma pessoa a cada 40 segundos. 0 suicídio encontra-se entre as dez principais causas de morte no mundo e entre as três primeiras quando considerada a faixa entre 15 e 44 anos de idade. Nas últimas quatro décadas, suas taxas aumentaram em $60 \%$, e nesses dados não estão inclusas as tentativas, que são 20 vezes mais frequentes do que os casos de suicídio consumados. Estima-se que, em 2020, o número de suicídios atinja 1,5 milhões de pessoas, avalia-se que ocorram 24 suicídios por dia. ${ }^{2}$

Os dados do Sistema de Informações sobre Mortalidade (SIM) mostram que os números de suicídio no Brasil vêm crescendo a cada ano, chegando a 9448 óbitos em 2010, com ênfase nas regiões sudeste e sul. Destaca-se a faixa etária entre 30 e 39 anos e, em relação a gênero, o número de suicídios entre os homens é três vezes maior que nas mulheres. ${ }^{3}$ No intuito de reforçar, o coeficiente médio de mortalidade por suicídio no Brasil, no período de 2004-2010 foi de 5,7\%. ${ }^{4}$ Nesse contexto, atentar para as tendências epidemiológicas relativas às tentativas e risco para o suicídio se constituem em passos importantes na elaboração de estratégias para a prevenção de comportamentos suicidas recorrentes ou até mesmo fatais. ${ }^{5}$

Em meio aos fatores de risco para suicídio pode-se citar a doença mental e física, o consumo excessivo de álcool e outras drogas, doenças crônicas, violência, mudanças repentinas e importantes na vida da pessoa, situação cultural e socioeconômica ou a combinação destes fatores. ${ }^{6}$ Quando há tentativa de suicídio, comumente essa pessoa é conduzida para uma instituição hospitalar mais próxima a ela e, na maioria das vezes, é atendida na unidade de emergência. Nesse cenário, são os profissionais de enfermagem que geralmente fazem o contato e ofertam os primeiros cuidados, no intuito de salvar vidas, devendo levar em consideração não somente o aspecto físico, como também os psicológicos envolvidos no processo. 


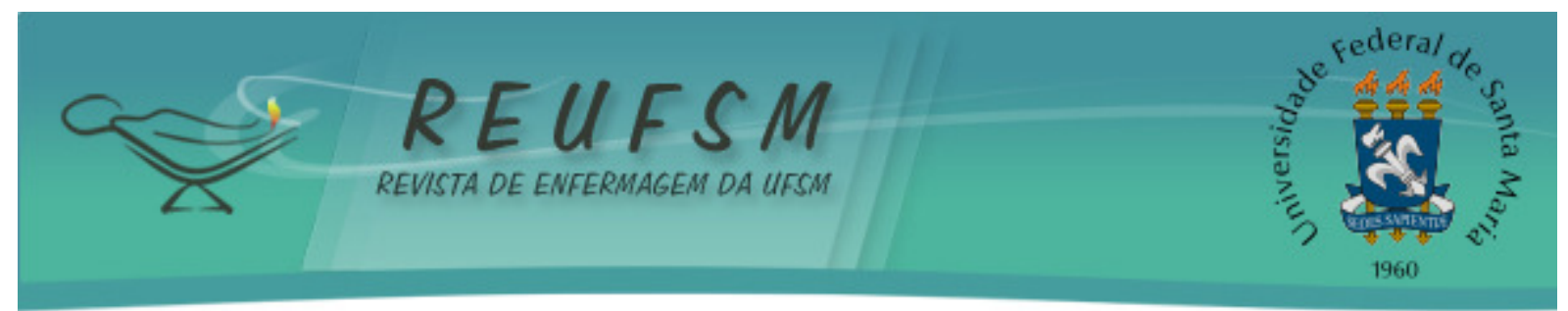

O profissional enfermeiro atuante no serviço de saúde deve estar qualificado e preparado para identificar as características que o paciente com potencial suicida apresenta, como pensamentos e atitudes que evidenciam desesperança, desespero e desamparo. Deve-se abordar o paciente de forma clara e cautelosa, mantendo a calma, empatia e abstendo-se das atitudes julgadoras. ${ }^{7}$

0 atendimento de emergência psiquiátrica, como em situações que envolvem comportamento suicida, consiste em ouvir atenciosamente, sem modular os sentimentos e ideias das pessoas que tentaram ou com ideação suicida. É importante que o profissional transmita segurança a elas, as acalme e colabore para que pensem e verbalizem sobre a pretensão de colocar sua própria vida em risco e o desejo de morrer. ${ }^{7}$ Para além disso, os cuidados contemplam a avaliação do estado emocional e do risco para o suicídio apresentado pela pessoa, o que, consequentemente, implicará na adoção de medidas de vigilância no sentido de evitar tentativas de suicídio. Ainda, ações com vistas a cuidar dos aspectos físicos que podem estar comprometidos em decorrência da tentativa de suicídio também são necessárias. ${ }^{8}$

Nesse sentido, a família tem papel relevante, tanto no apoio ao sujeito como fonte de informações e esclarecimentos referentes à tentativa de suicídio, visando um direcionamento do cuidado pela equipe prestadora de assistência à saúde. ${ }^{7} \quad$ Neste contexto, vale ressaltar a importância do apoio às famílias que vivenciam o comportamento suicida de um dos seus integrantes. 0 apoio prestado aos familiares é demonstrado de diversas formas pela equipe de enfermagem, como esclarecer dúvidas, prestar ou encaminhar o familiar para apoio psicológico, ofertar um ambiente que garanta privacidade e conforto, caracterizando assim um cuidado integral e humanizado. ${ }^{9}$

Os profissionais de enfermagem têm certas dificuldades em lidar com situações que envolvem tentativa e suicídio propriamente dito. A avaliação do comportamento suicida continua sendo um desafio para estes trabalhadores e, geralmente, ela é mais importante do que a compreensão da sua causa. ${ }^{10}$

Embora este estudo tenha como foco a equipe de enfermagem, considera-se que a atenção à população que possui comportamento suicida deva ser realizada por todos os profissionais que atuam nos serviços de saúde, incluindo a enfermagem. Nesse sentido, a atenção à pessoa nesses espaços engloba ações que conjugam saberes e trabalho de diferentes profissionais, com ênfase à multidisciplinaridade, cujas ações se somam e se complementam. ${ }^{11}$

Considerando o exposto, este estudo tem como questões norteadoras: Qual o conhecimento dos profissionais de enfermagem que atuam em um hospital geral de médio porte acerca do suicídio? Quais as intervenções realizadas por estes profissionais ao atender uma pessoa que possui comportamento suicida? Centrado nestas questões, 0 objetivo desta pesquisa consiste em analisar o conhecimento e as estratégias de intervenção de profissionais de enfermagem de um hospital geral, acerca do comportamento suicida.

\section{METODO}

Trata-se de uma pesquisa de natureza qualitativa e descritiva. A investigação ocorreu em um hospital geral, de médio porte, na região norte do Estado do Rio Grande do Sul, no período de julho a dezembro de 2012. A coleta de dados foi realizada com 10 profissionais de enfermagem, enfermeiros, técnicos e auxiliares de enfermagem que atuam nas três unidades de internação, clínica e cirúrgica, e na unidade de emergência do referido hospital. A recorrência nas informações identificadas nas entrevistas foi 


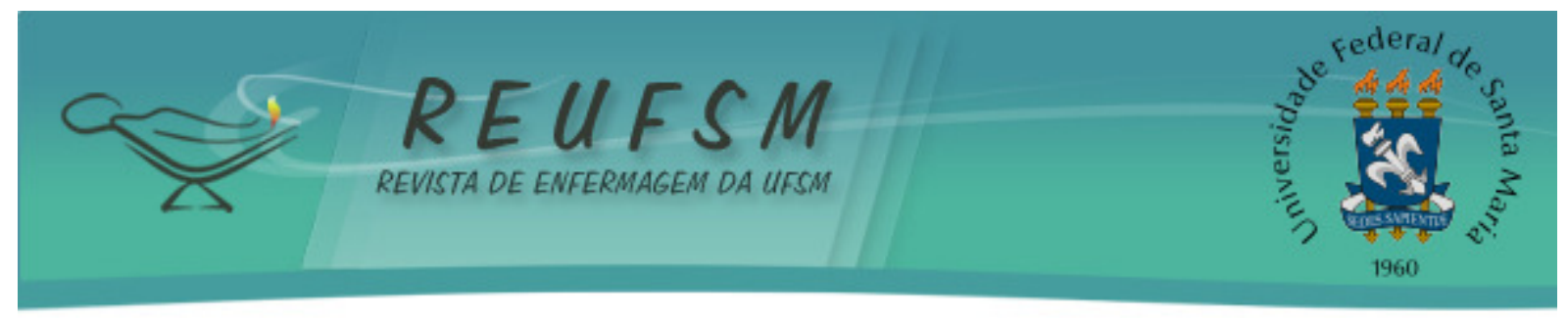

balizadora para o enceramento das mesmas e, assim, definir o número de entrevistados. 12

Dentre os critérios de inclusão foram considerados os profissionais vinculados ao serviço há pelo menos um ano, que prestam cuidados a pessoas pós-tentativa e/ou com risco para o suicídio. Foram excluídos os indivíduos que não se encaixaram nos critérios de inclusão ou que não manifestaram interesse em participar da pesquisa.

A obtenção dos dados ocorreu por meio de entrevista semiestruturada, contendo questões abertas e fechadas. A entrevista teve como questões norteadoras: Fale-me o que você sabe sobre suicídio? Como é para você atender uma pessoa que tentou ou com risco para o suicídio? Que cuidados você oferece para a pessoa que apresenta comportamento suicida?

As entrevistas foram gravadas em áudio-tape e posteriormente transcritas na íntegra, garantindo o anonimato com a codificação dos sujeitos pesquisados por meio de letras e números. A coleta de dados foi realizada na própria instituição hospitalar, de acordo com a disponibilidade do profissional de enfermagem entrevistado. A entrevista somente realizou-se após o aceite e assinatura do Termo de Consentimento Livre Esclarecido (TCLE).

Os dados foram analisados por meio de análise temática, dividida em três etapas: pré-análise, exploração do material, tratamento e interpretações dos dados obtidos. ${ }^{13}$

Os aspectos éticos foram respeitados, de acordo com a Resolução $n^{\circ} 196 / 96$, vigente na época em que o estudo foi realizado ${ }^{14}$ e o projeto de pesquisa foi aprovado pelo Comitê de Etica em Pesquisa da Universidade Federal de Santa Maria, mediante número do processo: 23081.004704/2011-65.

\section{RESULTADOS E DISCUSSÕES}

Dentre os participantes da pesquisa, quatro eram enfermeiros, cinco técnicos de enfermagem e um auxiliar de enfermagem. Do total, três eram masculinos e sete femininos. Sobre a idade, esta variou de 24 a 46 anos. Com relação ao tempo de atuação na instituição hospitalar, a maior parte dos profissionais desempenhava suas atividades de quatro a sete anos. Todos já atenderam pessoas com comportamento suicida no hospital.

A partir dos dados coletados no campo empírico da pesquisa sobre o conhecimento de profissionais de enfermagem e suas intervenções acerca do comportamento suicida, estes foram analisados à luz da literatura que discute essa temática. Os dados foram agrupados em dois temas, sendo que o primeiro versa sobre os motivos do suicídio na voz de profissionais de enfermagem e o segundo aborda as intervenções realizadas pela equipe de enfermagem junto a pessoas com comportamento suicida.

\section{Tema 1: Os motivos do suicídio na voz de profissionais de enfermagem}

A carência de informações por parte dos profissionais de saúde, incluindo a enfermagem, pode ocasionar descompasso entre as necessidades da pessoa que vivencia o risco ou apresentou tentativa de suicídio e as intervenções realizadas. ${ }^{15}$ Comumente, o comportamento suicida caracteriza-se como desfecho de sofrimento, presença de quadros nosológicos como: depressão, esquizofrenia, transtornos relacionados ao uso de substâncias e atos de desesperos, cuja detecção de sinais de desesperança e pedidos de ajuda podem passar desapercebidos pela equipe. ${ }^{15-16}$

Os participantes da pesquisa se reportaram a motivos que podem levar as pessoas a apresentarem comportamento suicida, evidenciados nas falas: 


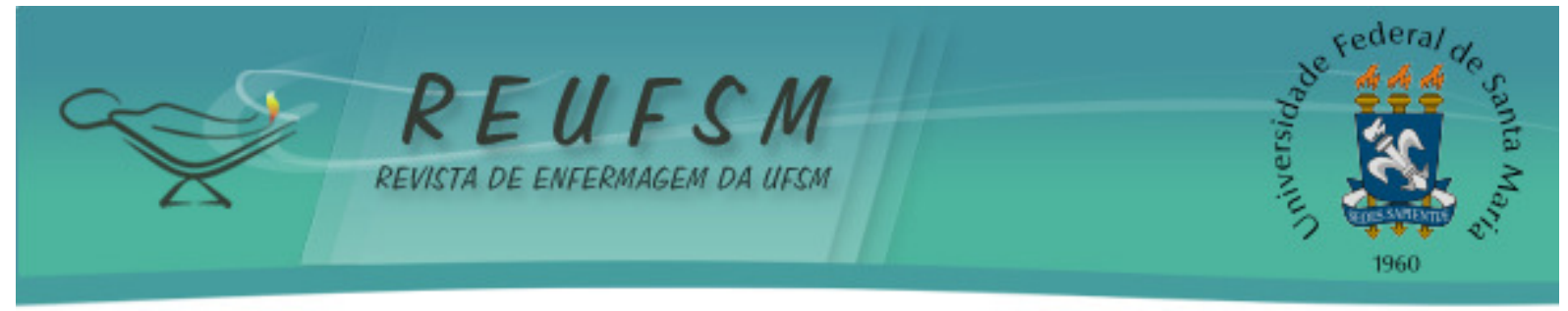

É um grande sofrimento que a pessoa tem e não consegue expressar esse sofrimento e acaba cometendo o suicídio. (E3)

As pessoas que cometem o suicídio têm algum problema de relacionamento, problema na família e tal, que não conseguem superar e cometem o suicídio. (E4)

As pessoas cometem quando não tem mais vontade de viver, por problemas familiares, dívidas, relacionamento, esse tipo de fatos. (E5)

Tem várias relações junto, familiar, profissional, que levam essa pessoa ao suicídio. (E7)

São problemas pessoais, família, relacionamento, as vezes morte de algum parente. (E8)

Também houve menção por parte dos profissionais de enfermagem de que o sofrimento leva o indivíduo a ter comportamento suicida, em que o suicídio se constitui na alternativa encontrada naquele momento para aliviar a situação de dor que está sendo vivenciada.

A pessoa não quer mais viver e acha uma forma de eliminar a sua vida. (E1)

Suicídio as pessoas cometem quando não tem mais vontade de viver. (E5)

É uma hora de desespero... em que a única solução para vida dela é a morte. (E6)

O acúmulo de angústias e aflições gera sofrimento emocional e algumas pessoas apresentam dificuldades de admiti-lo e enfrentá-lo, encontrando no suicídio uma forma de acabar com esse sofrimento. O desgaste emocional, associado ao preconceito, pode dificultar a identificação da necessidade de ajuda e/ou suporte especializado. ${ }^{17} \mathrm{Na}$ mesma linha de pensamento, estudo ${ }^{18}$ identificou que os profissionais de saúde não percebiam o comportamento suicida de pacientes que acessavam um serviço hospitalar como uma situação de crise que necessitava de ajuda.

A tentativa de suicídio habitualmente está associada à possibilidade de obter resultado no sentido de resolução dos problemas pessoais do indivíduo em sofrimento que, ao buscar o término de suas angústias, adota uma postura que vai contra a sua vida. ${ }^{19}$

A organização familiar desestruturada pode contribuir significativamente para incidência de planejamento suicida. ${ }^{20} \mathrm{~A}$ ausência de diálogo e divisão de responsabilidades dificultam a resolutividade de diversas circunstâncias negativas.

Outro aspecto elencado pelos pesquisados diz respeito à relação do suicídio com o adoecimento mental.

Se mata por algum problema psicológico, algum problema que não está legal. (E2)

Para mim geralmente são pessoas depressivas. (E4) 


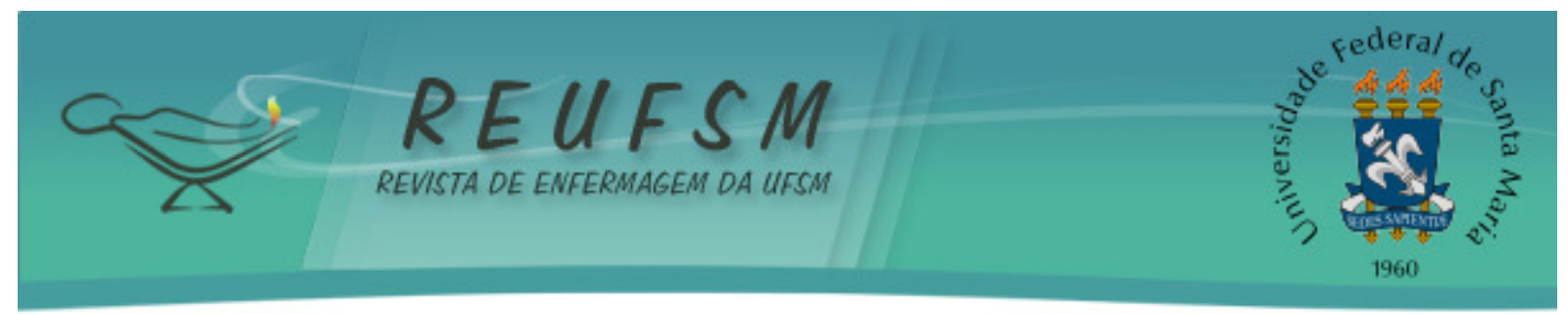

É uma fraqueza mental [...], leva a pessoa a depressão e aí ela tenta o suicídio, a pessoa cai na depressão, fica neurótica, daí ela tenta. (E8)

Estima-se que o risco de suicídio é de $4 \%$ em pacientes com transtornos do humor, $7 \%$ em dependentes de álcool, $8 \%$ em pessoas com transtorno bipolar e $5 \%$ em indivíduos com esquizofrenia. É importante ressaltar que o risco de comportamento suicida aumenta com a presença de comorbidades, uma vez que indivíduos com mais de um transtorno mental têm riscos expressivamente mais elevados de atentar contra sua vida. ${ }^{3}$

Sendo assim, a temática é considerada uma emergência em saúde, pois oferece risco de morte iminente, que carece da detecção dos fatores que, possivelmente, estejam associados a esse comportamento autoagressivo, a fim de reduzi-lo.

Neste estudo, evidencia-se que os profissionais de enfermagem se reportam aos motivos que podem levar as pessoas a apresentar comportamentos suicidas, parecendo ter clareza em relação a esses aspectos. Um dado importante a ressaltar é o fato de que os participantes da pesquisa não relacionam o suicídio exclusivamente à doença mental, mas sim a diversas situações cotidianas produtoras de sofrimento, o que corrobora com aquilo que é discutido na literatura que aborda essa temática.

\section{Tema 2: Intervenções realizadas pela enfermagem junto a indivíduos pós-tentativa e com risco para o suicídio}

Os profissionais de enfermagem entrevistados também se reportam aos cuidados às pessoas que tentaram suicídio, alegando que, muitas vezes, pelo fato de o paciente não aparentar o que está realmente sentindo, os cuidados não são prestados da maneira adequada, podendo comprometer a assistência de enfermagem.

Tem pessoas que conseguem, por exemplo, mascarar a tentativa de suicídio, na sua frente ela parece uma pessoa normal, que está com depressão, que está passando por um problema, mas que nunca vai tentar o suicídio... na sua frente ela pode ser uma pessoa e dizer que vai melhorar, que está tomando a medicação, está se cuidando e atrás de nós tudo acontece. (E1)

Já tivemos casos aqui que o paciente tentou o suicídio em casa, mas que não dava indício nenhum durante a internação, sinal nenhum de nada. (E3)

A gente nunca sabe quando a pessoa vai cometer o suicídio. (E5)

Alguns entrevistados citaram cuidados relacionados as questões clínicas decorrentes da tentativa de suicídio.

Assim, tomou uma medicação, primeira coisa que a gente faz é prestar os primeiros socorros, pegar um acesso venoso, uma sondinha, o médico vai te pedir alguma coisa, um lavado gástrico, conversar com a pessoa, se ela quiser te contar como foi. (E6)

Conversar, orientar, tentar ajudar, saber o problema dela, mas nem sempre a gente consegue, também fazer medicação que o médico prescreve e o que mais a gente faz é conversar mesmo, 


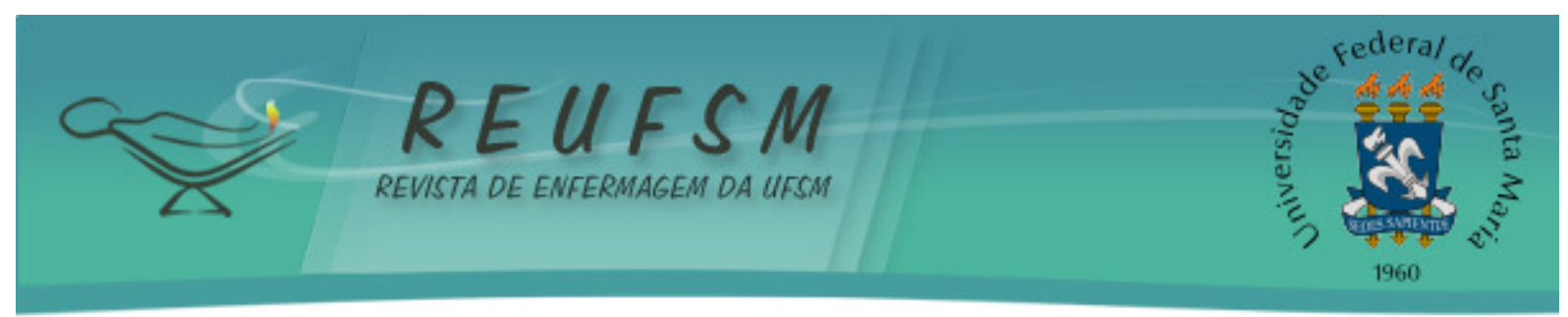

conversar com a família também, acalmar, porque sempre é um problema para eles. (E7)

Os hospitais precisam ter um ambiente calmo, tranquilo e seguro para assistir um paciente potencialmente suicida. É importante também registrar no prontuário e comunicar a equipe de enfermagem o risco para o suicídio, ter um tratamento implementado e é imprescindível trocar ideia com a equipe assistencial para decidir as melhores condutas a serem adotadas. ${ }^{7}$

0 atendimento a pessoas em crise suicida possui elementos básicos para uma melhor conduta. Em geral, os pacientes precisam ser ouvidos, o profissional deve ter a aceitação dos próprios sentimentos, incluindo a tolerância. Ainda, precisa ser um ponto de apoio, construir vínculo com o paciente, para que possa facilitar o tratamento e diminuir o risco para o suicídio. ${ }^{7}$

As intervenções da equipe de enfermagem frente a situações de risco para o suicídio consistem em acolher o paciente em local seguro para ambos, fazer a anamnese, o exame do estado mental, avaliar e classificar o risco para o suicídio, construir uma rede de apoio juntamente com serviços especializados, familiares/cuidadores e administrar terapia medicamentosa, se houver necessidade. 0 plano de tratamento deve ser flexível e passar por revisões periódicas.

Considerando as diversas possibilidades de atuação da equipe de enfermagem, é necessário que ela esteja devidamente qualificada para melhor identificar e compreender o paciente e sua família como um todo, adotando medidas assistenciais pertinentes à situação e estabelecer, assim, uma linha de cuidado humanizado.

Pensar em suicídio é se entregar a uma longa busca de porquês, que faz pensar e refletir sobre sentimentos, faltas, lacunas ou mistérios que rondam a existência daquele ser humano. Isso leva o profissional a questionamentos, como por exemplo, por que as pessoas se matam, o que aconteceu com aquela pessoa para desistir de viver e, consequentemente, remete a uma busca por respostas no sentido de aliviar a sensação de indignação e inconformismo, por alguém ter decidido acabar com sua própria vida. ${ }^{15}$

A partir dos discursos dos profissionais de enfermagem é possível organizar e planejar ações de educação em saúde, com o propósito de qualificar a práxis de enfermagem junto à pessoa que tentou ou está com risco para o suicídio. Assim sendo, a constante qualificação, capacitação e busca pelo conhecimento sobre o tema suicídio e seus fatores relacionados possibilitam à equipe de enfermagem realizar um atendimento mais eficaz a esses indivíduos. Além disso, a interlocução da equipe de enfermagem do hospital com os demais serviços da rede de atenção em saúde mental pode se constituir em estratégia para a construção de um plano de cuidados intersetorial ${ }^{21}$ às pessoas com risco ou que tentaram suicídio no momento da alta hospitalar. Ainda, a articulação entre os profissionais dos diferentes serviços contribui para o desenvolvimento de práticas de cuidado integrais ${ }^{16}$ a esse contingente populacional, com vistas a minimizar o sofrimento emocional decorrente de ideação ou tentativa de suicídio.

\section{CONSIDERAÇÕES FINAIS}

O presente estudo mostrou que o suicídio é entendido pelos profissionais de enfermagem como um meio de fuga da realidade, relacionado ao sofrimento e dificuldades cotidianas e decorrente de adoecimento mental, ao mesmo tempo em que representa a resolução de problemas de ordem pessoal, familiar e psicológica. Evidenciou a importância das equipes de enfermagem atuantes em hospitais gerais na identificação de comportamentos suicidas e de fatores que exacerbam o risco de suicídio, bem como a 


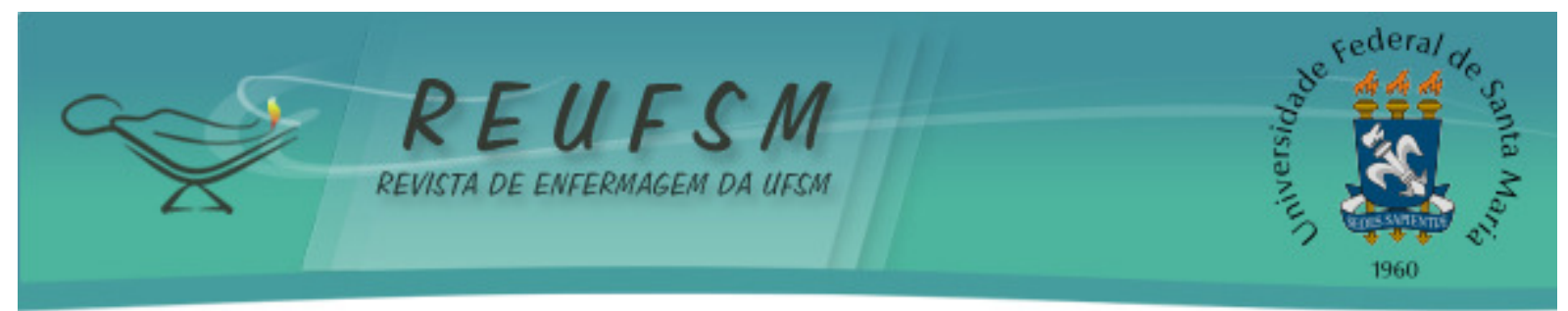

utilização de estratégias para a sua prevenção. Ainda, destacou a relevância de intervenções de enfermagem junto às pessoas que tentaram suicídio, no sentido de proporcionar segurança e confiança ao paciente, família e instituição de saúde.

Sendo assim, os profissionais de enfermagem devem estar qualificados para realizar as intervenções necessárias ao paciente que procura o serviço e para fornecer o encaminhamento mais adequado a cada caso. Ainda, o estabelecimento de um bom relacionamento entre profissional e paciente pode ser relevante na prevenção de tentativas de suicídio. Contudo, é importante destacar que há vários elementos inerentes à subjetividade do sujeito que influenciam na presença de comportamento suicida e na própria adesão do tratamento, que não podem ser desconsiderados pela equipe.

Não obstante, identifica-se que os profissionais, participantes do estudo, apresentam dificuldades em lidar com situações que envolvam comportamento suicida, o que, provavelmente, tem relação com o pouco conhecimento acerca dessa temática, o que indica a necessidade de fortalecer as discussões sobre essa temática. Quanto maior o conhecimento acerca do tema, maiores as possibilidades de implantação e aprimoramento de intervenções que visam a redução de danos, além do bem estar desses indivíduos.

Por outro lado, não se pode relacionar a qualidade das intervenções unicamente à qualificação da equipe de enfermagem, até porque, muitas vezes, a presença de comportamento suicida em pacientes assistidos persiste apesar do cuidado prestado. Desse modo, se houve uma avaliação precisa e planejamento das ações de enfermagem baseado nas necessidades do sujeito, os profissionais poderão tranquilizar-se e ter noção de que algumas situações ultrapassam a sua governança.

Com base no exposto, destaca-se a relevância do tema e a necessidade de realização de novos estudos, para fornecer maior embasamento às ações dos profissionais na abordagem aos pacientes que tentaram suicídio e que apresentam risco para o mesmo.

\section{REFERÊNCIAS}

1. Carvalho A, Peixoto B, Saraiva CB, Sampaio D, Amaro F, Santos JC, et al. Plano nacional de prevenção do suicídio 2013/2017. Portugal: Direção-Geral da Saúde, Programa Nacional para a Saúde Mental; 2013.

2. World Health Organization (WHO). Preventing suicide: a global imperative. World Health Organization; 2014 [acesso em 2015 mar 24]. Disponível em: http://apps.who.int/iris/bitstream/10665/131056/1/9789241564779_eng.pdf.

3. Brasil. Ministério da Saúde. Sistema de Informações sobre Mortalidade (SIM). Brasília (DF): Ministério da Saúde; 2014.

4. Marin-Leon L, Oliveira HB, Botega NJ. Suicide in Brazil, 2004-2010: the importance of small counties. Rev Panam Salud Publica [Internet]. 2012 [acesso em 2015 abr 23];32(5):351-9. Disponível em: http://www.scielosp.org/pdf/rpsp/v32n5/v32n5a05.pdf.

5. Ting SA, Sullivan AF, Boudreaux ED, Miller I, Camargo Jr AC. Trends in US emergency department visits for attempted suicide andself-inflicted injury, 1993-2008. Gen Hosp Psychiatry [Internet]. 2012 [acesso em 2015 abr 22];34(5):557-65. Disponível em http://www.ncbi.nlm.nih.gov/pmc/articles/PMC3428496/.

6. Organização Mundial da Saúde (OMS). Saúde pública ação para a prevenção de suicídio: uma estrutura. Organização Mundial da Saúde; 2012. 


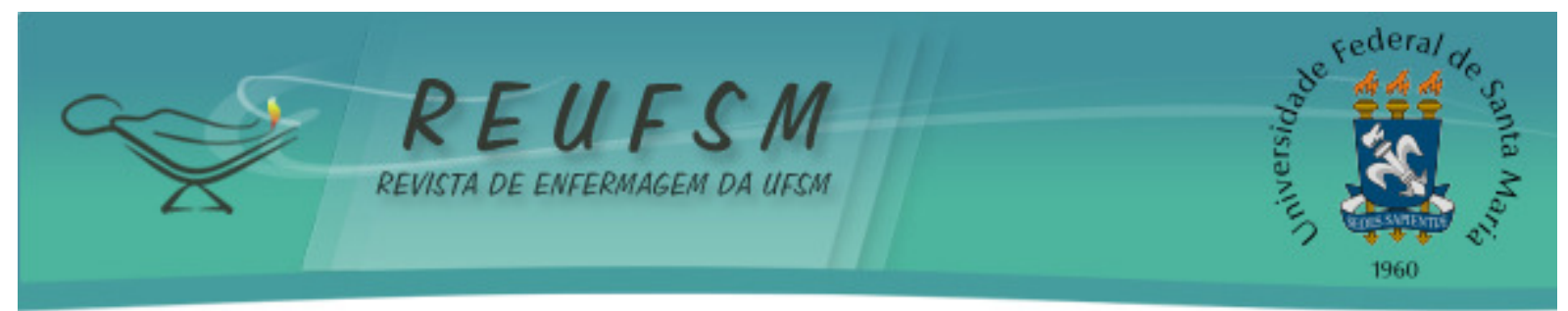

7. Bertolote JM, Mello-Santos C, Botega NJ. Detecção do risco de suicídio nos serviços de emergência psiquiátrica. Rev Bras Psiquiatr [Internet]. 2010 [acesso em 2014 set 18];32 Supl 2:S87-S95. Disponível em: http://www.scielo.br/pdf/rbp/v32s2/v32s2a05.pdf.

8. Rodrigues AA, Kapczinski F. Risco de suicídio. In: Quevedo J, Carvalho AF, organizadores. Emergências psiquiátricas. $3^{\text {a }}$ ed. Porto Alegre: Artmed; 2014.

9. Buriola AA, Arnauts I, Decesaro MN, Oliveira MLF, Marcon SS. Assistência de enfermagem às famílias de indivíduos que tentaram suicídio. Esc Anna Nery [Internet]. 2011 [acesso em 2014 out 10];15(4):710-6. Disponível em: http://www.scielo.br/pdf/ean/v15n4/a08v15n4.pdf.

10. Ores LC, Quevedo LA, Jansen K, Carvalho AB, Cardoso TA, Souza LDM, et al. Risco de suicídio e comportamentos de risco à saúde em jovens de 18 a 24 anos: um estudo descritivo. Cad Saúde Pública [Internet]. 2012 [acesso em 2014 out 17];28(2):305-12. Disponível em: http://www.scielo.br/pdf/csp/v28n2/09.pdf.

11. Gutierrez BAO. Assistência hospitalar na tentativa de suicídio. Psicologia USP [Internet]. 2014 [acesso em 2015 abr 21];25(3):262-9. Disponível em: http: //www.scielo.br/pdf/pusp/v25n3/0103-6564-pusp-25-03-0262.pdf.

12. Turato ER. Tratado de metodologia da pesquisa clínico-qualitativa: construção teóricoepistemológica, discussão comparada e aplicação nas áreas da saúde e humanas. $3^{\mathrm{a}}$ ed. Petrópolis/Rio de Janeiro: Vozes; 2008.

13. Minayo MCS, organizadora. Pesquisa social: teoria, método e criatividade. $29^{\mathrm{a}}$ ed. Petrópolis: Vozes; 2010.

14. Brasil. Ministério da Saúde. Conselho Nacional de Saúde. Resolução CNS n 196 , de 10 de outurbo de 1996. Aprova diretrizes e normas regulamentadoras de pesquisas envolvendo seres humanos. Brasília (DF): Ministério da Saúde; 1996 [acesso em 2011 dez 10].

Disponível em: http://conselho.saude.gov.br/resolucoes/1996/Reso196.doc.

15. Barbosa FO, Macedo PCM, Silveira RMC. Depressão e o suicídio. Rev SBPH [Internet]. 2011 [acesso em 2014 out 17];14(1):233-43. Disponível em: http://pepsic.bvsalud.org/pdf/rsbph/v14n1/v14n1a13.pdf.

16. Lima DD, Azevedo RCS, Gaspar KC, Silva VF, Mauro MLF, Botega NJ. Tentativa de suicídio entre pacientes com uso nocivo de bebidas alcoólicas internados em hospital geral. J Bras Psiquiatr [Internet]. 2010 [acesso em 2014 out 20];59(3):167-72. Disponível em: http://www.scielo.br/pdf/jbpsiq/v59n3/a01v59n3.pdf.

17. Fontenelle P. Suicídio: o futuro interrompido: guia para sobreviventes. São Paulo: Geração; 2008.

18. Vidal CEL, Gontijo ED. Tentativas de suicídio e o acolhimento nos serviços de urgência: a percepção de quem tenta. Cad Saúde Coletiva [Internet]. 2013 [acesso em 2015 abr 21];21(2):108-14. Disponível em: http://www.scielo.br/pdf/cadsc/v21n2/02.pdf.

19. Heck RM, Kantorski LP, Borges AM, Lopes CV, Santos MC, Pinho LB. Ação dos profissionais de um centro de atenção psicossocial diante de usuários com tentativa e risco de suicídio. Texto \& Contexto Enferm [Internet]. 2012 [acesso em 2014 nov 5];21(1):26-33. Disponível em: http://www.scielo.br/pdf/tce/v21n1/a03v21n1.

20. Baggio L, Palazzo LS, Aerts DRGC. Planejamento suicida entre adolescentes escolares: prevalência e fatores associados. Cad Saúde Pública [Internet]. 2009 [acesso em 2014 nov 6];25(1):142-50. Disponível em: http://www.scielo.br/pdf/csp/v25n1/15.pdf. 


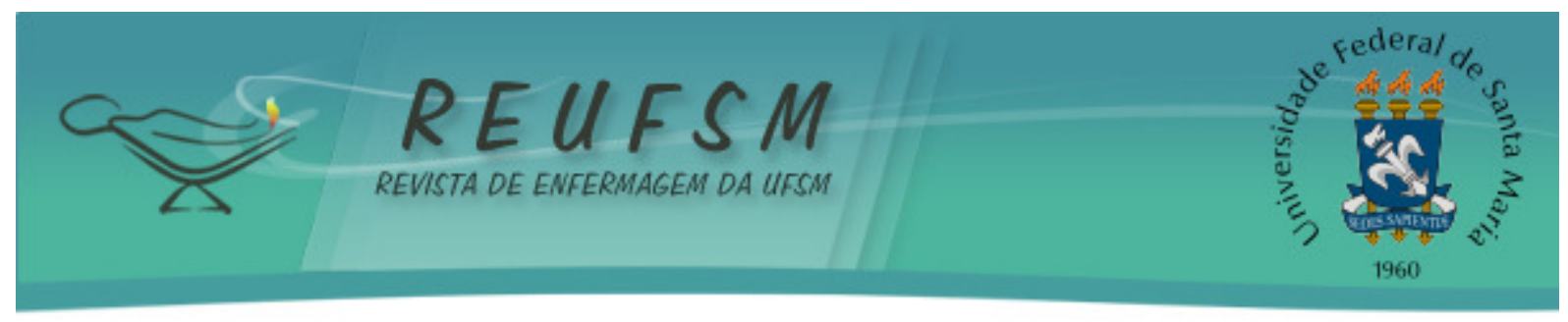

21. Azevedo EB, Carvalho RN, Cordeiro RC, Costa LFP, Silva PMC, Ferreira Filha MO. Tecendo práticas intersetoriais em saúde mental para pessoas em sofrimento psíquico. Rev Enferm UFSM [Internet]. 2014 [acesso em 2015 jan 20];4(3):612-23. Disponível em: http://cascavel.ufsm.br/revistas/ojs-2.2.2/index.php/reufsm/article/view/13562.

Data de recebimento: 20/01/2015

Data de aceite: 18/05/2015

Contato do autor responsável: Leila Mariza Hildebrandt

Endereço postal: Rua dos Carajás, 82 - Bairro Pindorama - 98700-000 - Ijuí/RS

E-mail: leilahildebrandt@yahoo.com.br 